\title{
Fatigue Tests - Important Part of Development of New Vehicles
}

\author{
Miloslav Kepka ${ }^{1,}$, Miloslav Kepka Jr. ${ }^{1}$, Pavel Zlabek ${ }^{1}$, Petr Heller ${ }^{1}$, Jan Chvojan $^{1}$, Vaclav Mentl ${ }^{1}$ et al. \\ ${ }^{1}$ University of West Bohemia, Faculty of Mechanical Engineering, Univerzitni 8, 30614 Pilsen, Czech Republic
}

\begin{abstract}
In city of Pilsen (Czech Republic) modern transport engineering is developed. The Skoda Transportation (production company) has successfully been producing rail and road vehicles for many years (electric locomotives, trams, metro cars, trolleybuses, battery buses). This producer cooperates in developing these vehicles with the Research and Testing Institute (commercial research institute) and with the University of West Bohemia (public university). Fatigue tests are carried out by the Dynamic Testing Laboratory at the Research and Testing Institute and by the Regional Technological Institute, the research center of the Faculty of Mechanical Engineering at the university. The paper describes various fatigue tests and presents their practical realization in the mentioned laboratories.
\end{abstract}

\section{Introduction}

Fatigue tests can be classified with respect to various criteria. For instance, the following sorting is a common one:

- by the size of the structure tested (test specimen of a material, structural node, vehicle substructure or component, and the entire vehicle);

- by the controlling quantity (force, displacement, strain, acceleration, temperature);

- by the number of controlling quantities (a single controlling quantity, multiple controlling quantities);

- by the number of load directions (uniaxial loading vs. multi-axial loading);

- by the load-time profile (harmonic profile, a range of harmonic cycle blocks, a random process).

Fatigue tests may, however, be classified in other ways as well, depending on their role in research and development:

- tests intended to provide new fundamental scientific findings in the field of fatigue of materials and structures;

- tests intended to provide input data for expert reports and fatigue life calculations;

- tests mapping the impact of metallurgical production or subsequent processing upon final fatigue strength and life;

- tests conducted as part of a product development process (designers typically use these to validate design assumptions, results of computations, and computational models);

- prescribed fatigue tests (unless these are completed successfully, the product must not be introduced to the market or put into service);

- tests aimed to identify and remove causes of fatigue failures in service.
A special case of fatigue testing are virtual fatigue tests. Various computational programs for multi-body simulation, finite element method and dynamics are used to implement these virtual tests. A suitable fatigue postprocessor is necessary for this type of activity.

The most of the listed types of fatigue tests apply to the development of rail and road vehicles. The paper (and the poster) presents interesting examples of fatigue tests, which have been performed in collaboration of the Skoda Transportation (production company) with laboratories of the Research and Testing Institute and the Regional Technological Institute.

The authors of this paper have worked in the Skoda Transportation or the Research and Testing Institute in recent years, and today they continue to collaborate with these companies as academic or university researchers.

\section{Rail vehicles}

\subsection{Activities of Research and Testing Institute}

In city of Pilsen there is a long-standing tradition of testing the undercarriage frames of rolling stock. The Research and Testing Institute has a dominant position in this field and provides these services to domestic and foreign customers. Some examples are in Fig. 1.

A majority of bogie frames are made by welding or fabricated as a combination of cast parts and weldments. They are complex and intricate-shaped structures with a number of geometric notches and weakened locations. In general, boogie frames are heavy-duty parts of railway vehicles whose operation must be trouble-free.

Bogies operate under severe static, quasi-static and dynamic loads and their service failure would threaten many lives and lead to material losses. 
Hence, the development, manufacturing and testing of bogies demands maximum attention from the outset, through strength calculations and optimization of the structural design, to the final experimental verification.

Prior to the fatigue test, stresses in the bogie frame under static loads are experimentally analysed. Exceptional loads, service loads and partial service loads are the load categories for which the stress and deformation responses are measured and evaluated.

Fatigue tests of bogie frames of railway vehicles fall into the group of prescribed tests. The objective of the prescribed procedures is to demonstrate that the structure does not sustain permanent deformation under exceptional or service loads and that no cracks form during fatigue testing. The fatigue test simulates all major forces, which are caused in service by the vehicle's mass, track irregularities, the vehicle's running curves, acceleration, braking, etc.

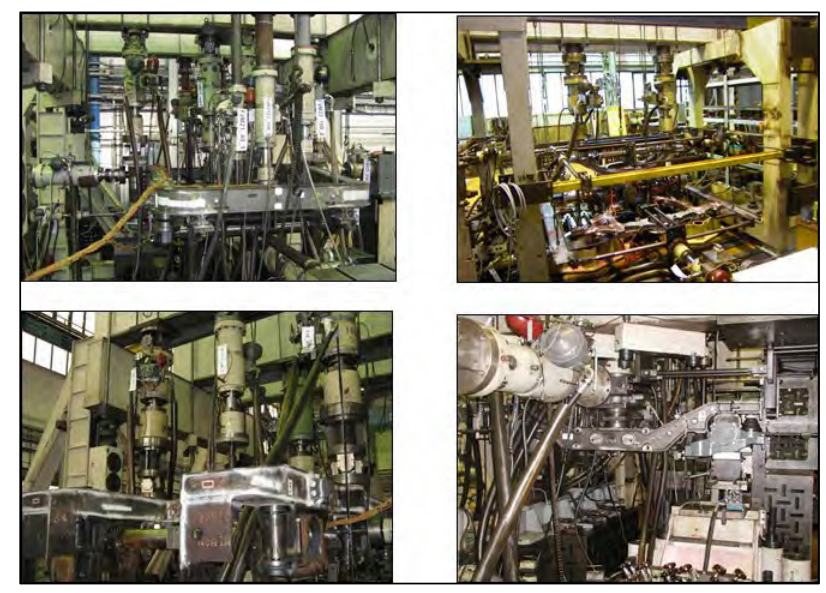

Fig. 1. Fatigue tests of bogies and undercarriage frames.

With the development of the testing technology, able to combine individual control electronic channels of hydraulic load actuators to multichannel units, began to fulfill dreams of some engineers about an experimental thorough assessment of all the operational and exceptional loading impacts on the bogie frames design. Similarly, as on their computational model.

With the development of the test technique, the dreams of some engineers have begun to perform a thorough laboratory simulation of all operational and exceptional loads that affect the bogie frame in operation. Similarly, as on their computational model.

All accelerations of bogie weights like motors, gear boxes, brake units etc. should by physically simulated in all orthogonal directions. The number of loading actuators rises up (for example up to twenty-two). The authors have already discussed within the professional public increasing customer demands for the number of load forces.

The complications associated with this phenomenon cannot be overlooked. It is relatively easier to compute a detailed part of the bogie frame, but practically much more complicated. Each actuator has to have minimum space around itself.

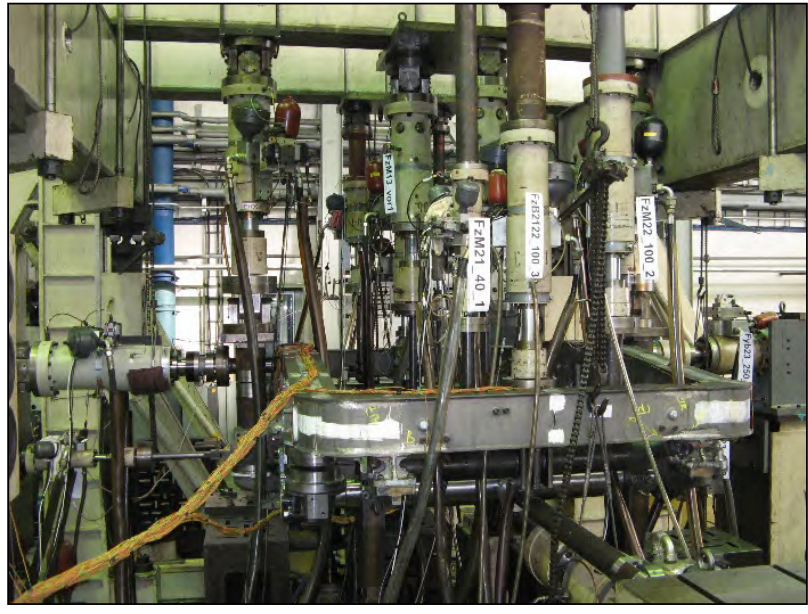

Fig. 2. Test of locomotive frame with twenty-two actuators.

The complicated fatigue test consists many procedures and partial tasks like test stand design, construction of appropriate fixture parts and their manufacturing, assembly of the test stand, technical preparation - installation of strain gauges and other sensors, performing static tests, measurement of all required variables, their analysis and evaluation, cycling etc. Fixture parts and their assembling for the connection for a "special" force introducing can be difficult, complicated and challenging, the impact of this force on the fatigue strength of the bogie frame negligible. It is the key and experienced decision of designers if they want to use - simpler test stand or more complicated. Both can be good and both can be wrong. Examples of tests with a radically different number of load forces are in Fig. 2 and Fig. 3.

\subsection{Activities of Regional Technological Institute}

The university research center focuses more on research and development, but strength and fatigue tests are also an integral part of these activities.

The development of tramway bogie for narrow track gauge is one of the interesting project. A simplified fatigue test under the relevant UIC directive was also part of the development process. The tram bogie was subjected to four decisive forces in this test. A photo of the test is in Fig. 3.

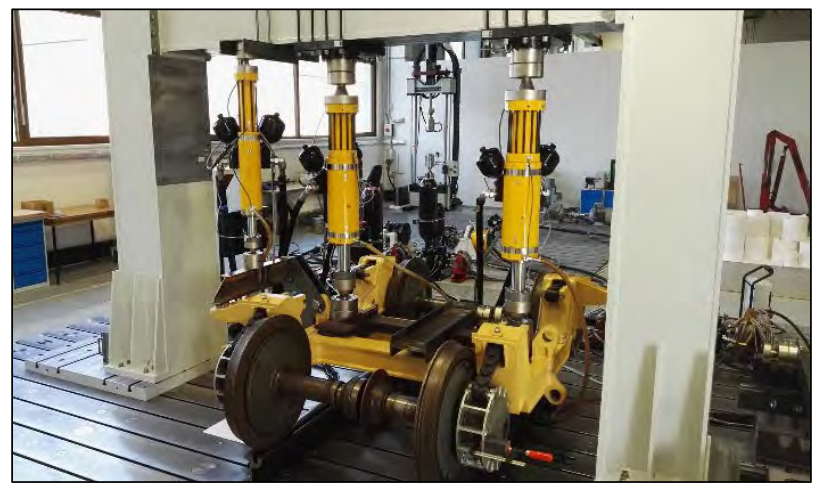

Fig. 3. Test of tram bogie with four actuators. 
The next interesting projects of the Regional Technology Institute was the development of the hybrid body of a metro car. The "political" idea of the project was a successful demonstration that even technical university can transform own expertise to a functional product and can manage this type of project.

As a demonstrator, the real sample of the hybrid body was designed, manufactured and tested. This body was made of a higher strength steel and special composite panels. The design was supported by FEM calculations, fundamental research of mechanical properties of all used materials. The fatigue tests of important structural details were organised in collaboration with the Research and Testing Institution. Fig. 4 shows testing the node connecting composite sides and bottom part of body. A photo of the manufacture of the body is in Fig. 5. Static strength tests of the whole body according to the relevant standards were realised in testing hall of the Skoda Transportation. The photo of the test is in Fig. 6.

The potential for weight saving of $800 \mathrm{~kg}$ was confirmed. The body has lower weight and manufacturing labour more intensive than the existing one. Thus, it can save production and operating costs. Some technologies was patented during the solution of the project.

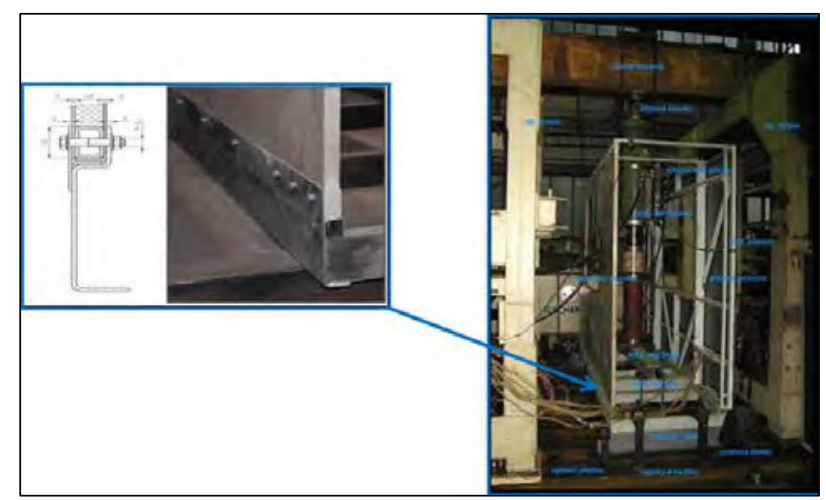

Fig. 4. Fatigue test of the node connecting composite sides and bottom part of body.

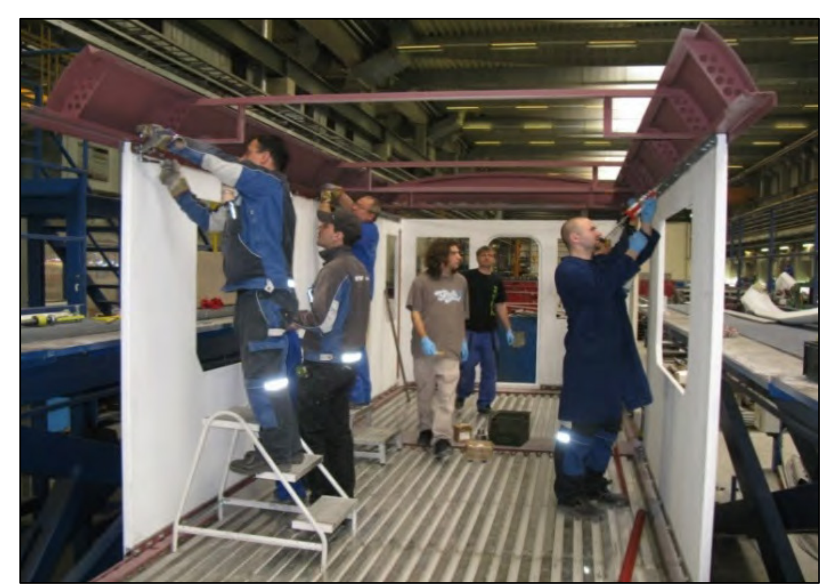

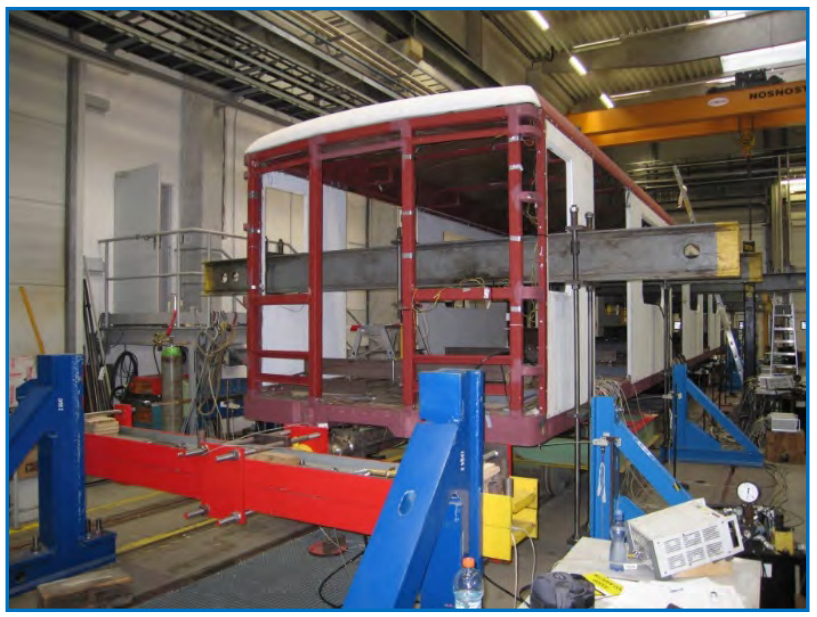

Fig. 6. Static strength test of hybrid body.

\section{Road vehicles}

\subsection{Activities of Research and Testing Institute}

City of Pilsen also has a long-standing tradition of testing bodyworks and undercarriage parts of road vehicles for urban public transport. The Research and Testing Institute provides for various domestic manufacturers not only fatigue tests, but also dynamic calculations, strength calculations by finite element method, measurement of operational stresses on model and real (city) routs.

The original methodology was applied at development of series of Skoda buses (mainly trolleybuses), Fig. 7.

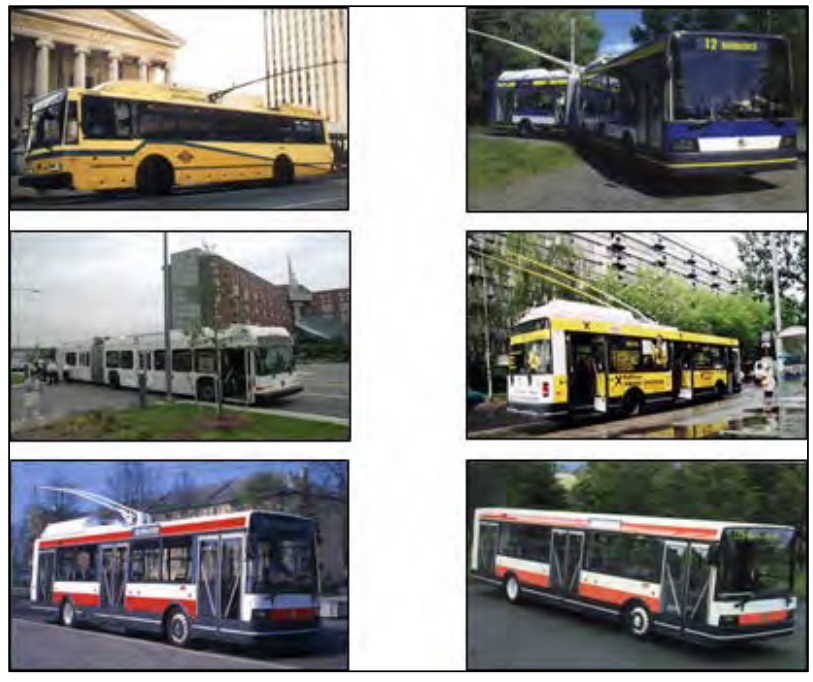

Fig. 7. Skoda trolleybuses supported by broad fatigue testing.

The methodology, which has been successfully applied for many years in the development of Skoda trolleybuses and buses, has been presented in the literature in the past. This methodology is now being

Fig. 5. Manufacture of hybrid body. 
developed in connection with the production of battery buses.

The relationship between testing vehicles on test tracks and on a test stand is schematically illustrated in Fig. 8.

At the Research and Testing Institute, it is possible to test whole vehicles. Stand tests are an important part of the process of optimizing the mechanical structure of the vehicle. Testing complete bodywork on a test stand is an efficient method for choosing the best design with regard to the desired operational strength and fatigue life.

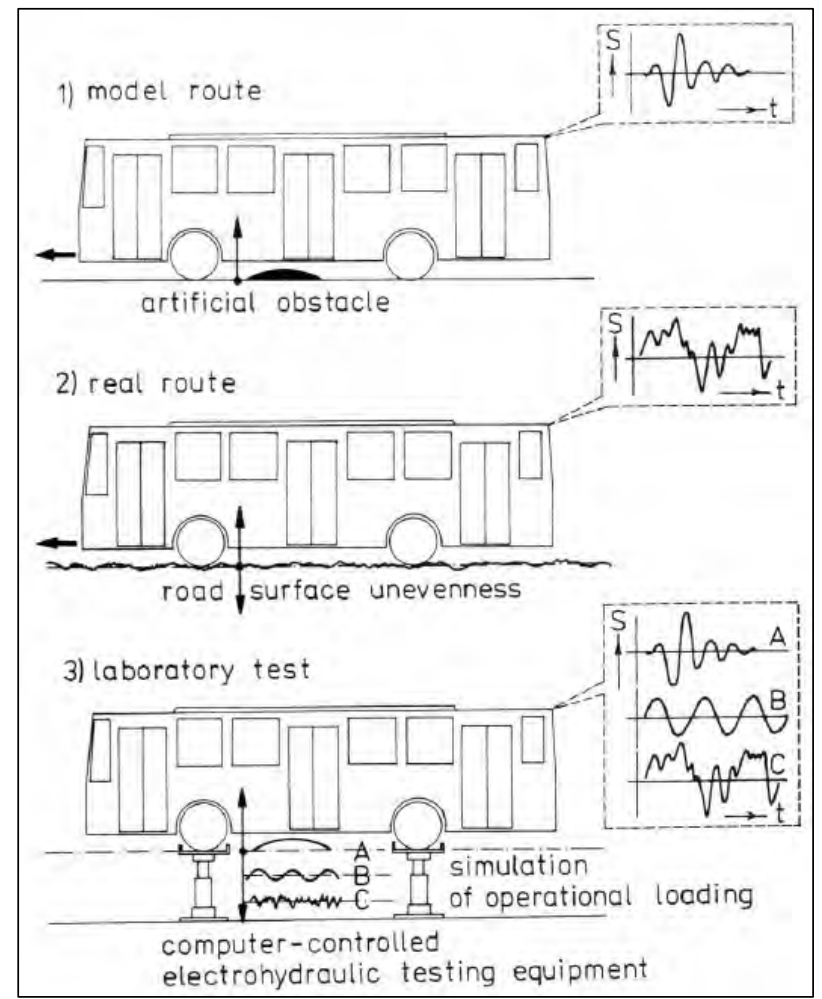

Fig. 8. Testing vehicles on test tracks and on test stand.

The design variants to be compared are usually prepared with a support of computational methods. The experimental programme on the test stand is also a test on the production technology and quality of labour. The test stand at the Research and Testing Institute is a computer-controlled electro-hydraulic device, consisting of several independent excitation cylinders where the test object is mounted for test. This sophisticated equipment ensures great flexibility in terms of test programmes and, at the same time, absolute reproducibility of the tests.

Experiments and tests on the stand are aimed at investigating the stress response in tested body related to excitation forces or deformations applied to the wheels. Three typical test modes are available: static, dynamic, dynamic-strength.

Static tests are used to determinate the stress fields and changes in the body resulting from torsion deformation from the up and down movement of individual wheels.
Dynamic tests use harmonic excitation whereby both acceleration and mechanical stresses are monitored at selected points on the body, response frequencies are determinated and parameters of the damping elements (such as bellow, dampers and tyres) are tested.

Dynamic-strength test are used to determinate the stress response to shock experienced in situations where the vehicle rides over a bump. The test stand makes it possible to simulate a driving on a real road characterized by random distribution of obstacles of a given statistical nature.

Fig. 9 is an example of possibilities of operational load simulations on test stands: simulation of vertical road unevenness and simulation of force acting in connection between the front and rear parts of the articulated vehicle.

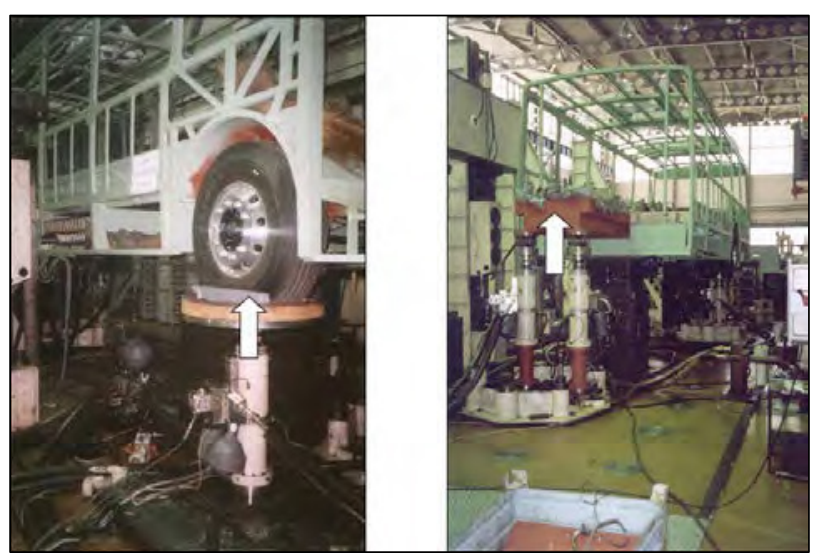

Fig. 9. Possible simulations of operational loading on test stand.

Fig. 10 shows the most complex test performed on test site of the Research and Testing Institute. The construction of the body and undercarriage frame of the first fully low-floor articulated Skoda trolleybus was very complex tested. The operation of this trolleybus showed absolute reliability in terms of strength and fatigue life.

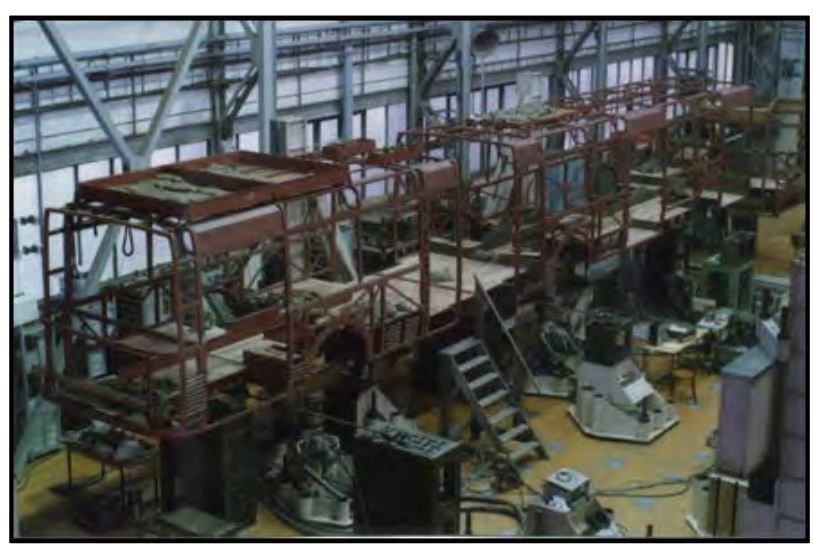

Fig. 10. Structure of articulated trolleybus on test stand.

In order to get a comprehensive view on the future operational reliability of the vehicle, it is necessary to deal with the detailed investigation of the fatigue 
strength and lifetime of the important construction nodes and vehicle components.

Laboratory tests on important parts and subassemblies aim at choosing the most advantageous design and technology with regard to the desired operational strength and lifespan of the whole body. Tests and experiments are typically performed on parts and their configurations that appear frequently in the design, e.g. joints between supporting sections in the body, or on parts exposed to extreme stress during operation (front spring holders, rear axle spring holders, articulated coupling between twin coaches, etc.)

During the tests, the decisive operational stresses are simulated by means of electro-hydraulic tests equipment. Parts to be tested are geared with strain gauges and mounted on the test stand. Measured data are subsequently used to analyse stress during laboratory tests and establish limit value states decisive for the fatigue strength and lifespan of the parts. By exposing different design variants to the same test conditions, it is possible to choose the best solution for the given operating environment.

Careful planning of the test programme, including in interface conditions for parts under test, is essential for establishing straightforward relationships between the test and real operating conditions.

The laboratory tests can be divided into two main groups: static tests and tests of fatigue strength and fatigue life.

Static tests consist of detailed analysis of the stress and deformation fields relating to the given test conditions.

Fatigue tests are used to establish zones and points of critical stress in the construction, i.e. points where fatigue may cause early failures.

The measured data are used for deriving various reliability parameters of the design by calculation. Various fatigue test methods are available (some possibilities are schematically illustrated in Fig. 11.) with different requirements as to the information on the real operational stresses, testing times, number of samples and overall costs.

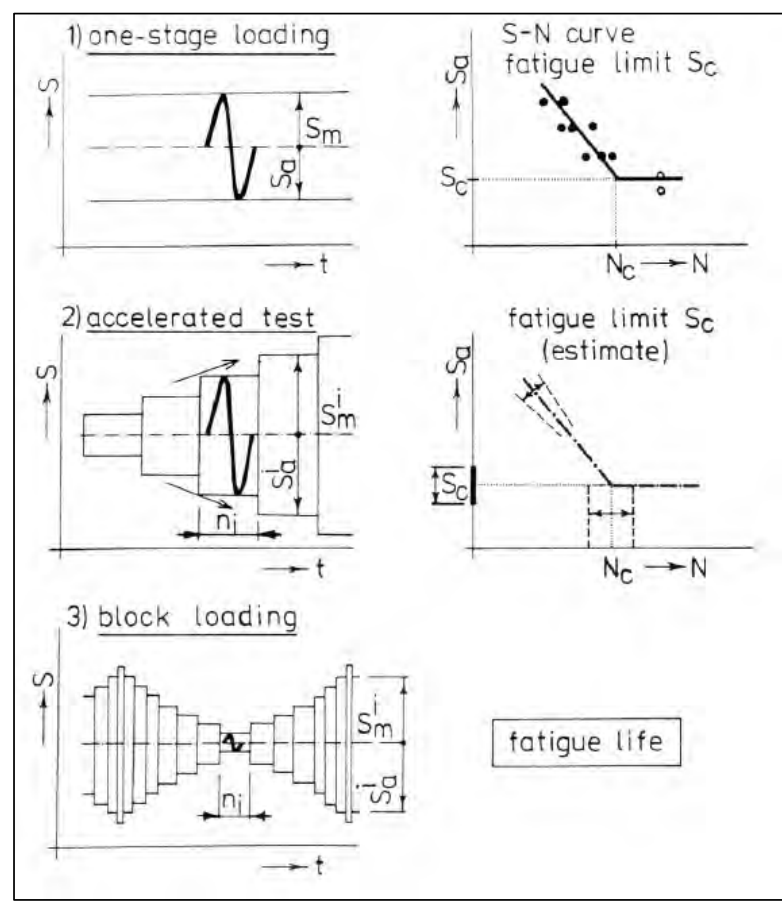

Fig. 11. Industry-friendly test load layout for fatigue testing of vehicle components.

The classic fatigue test with harmonic loading is used to derive the S-N curve and the fatigue limit of the part under test.

The accelerated fatigue test, where the complex stress succession in increased in steps, may be used in cases where the results, e.g. the fatigue limits for given critical point, are needed urgently and where poorer accuracy of information is acceptable. Compared to the classic fatigue tests, the accelerated methods of testing are faster, less costly and require fewer test samples.

The test on operational fatigue life uses a test programme based on a stress profile determinated in the real operating conditions of the vehicle. Thus, it is possible to derive very realistic estimates of the lifespan for a chosen critical part of the body. Disadvantages of this method are: high cost, long testing time and lesser general, applicability of the results.

The laboratory tests of critical vehicle parts yield the following information:

- fatigue limit for given critical point in a part/subassembly,

- response characteristics in the above points measured during test runs of vehicles on the both model and real test tracks.

In evaluating, the following criteria can be observed:

- maximum stress amplitudes are within the range of infinite fatigue life: results satisfactory, no changes in design necessary,

- maximum stress amplitudes exceed significantly the fatigue limit: results unsatisfactory, the design must be improved and the changes verified experimentally,

- maximum stress amplitude are in a region of the fatigue limit: further analysis is needed to assess the effect of the real service conditions on the given critical points, with regard to the lifespan. 
Many of the laboratory tests were precisely planned and implemented during the development of Skoda trolleybuses. Examples are in Fig. 12 to Fig. 15.

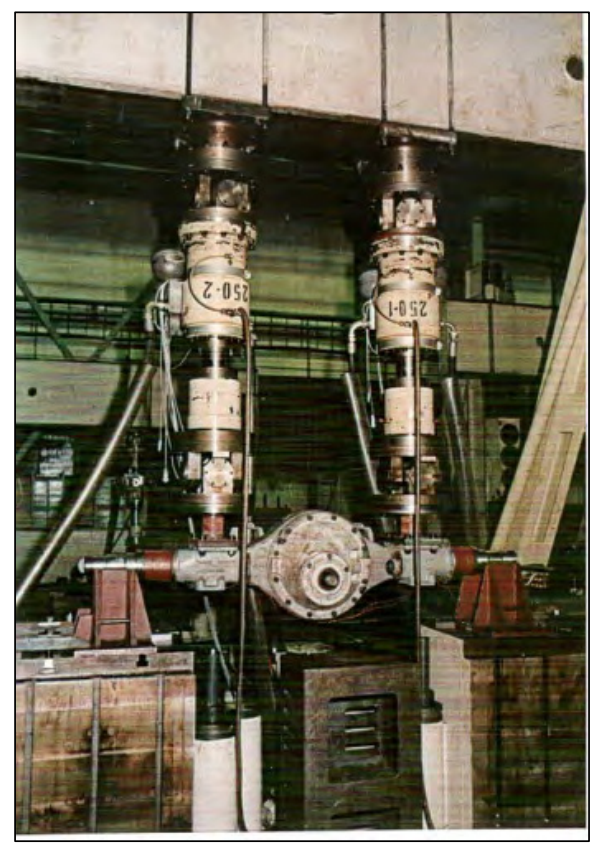

Fig. 12. Fatigue test of a rear axle.

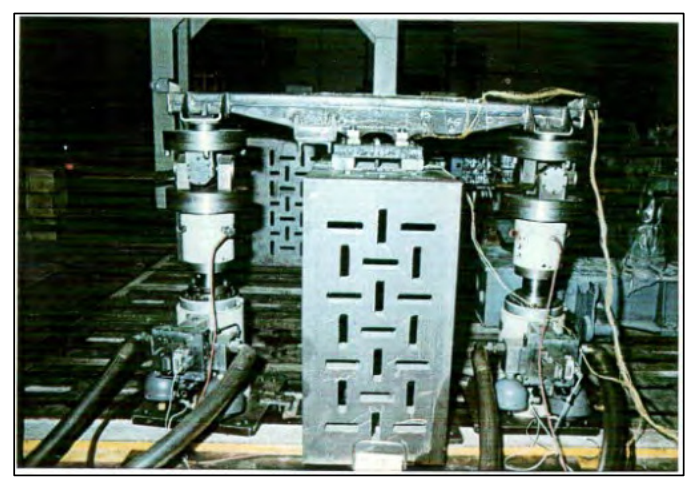

Fig. 13. Fatigue test of a rear suspension beam.

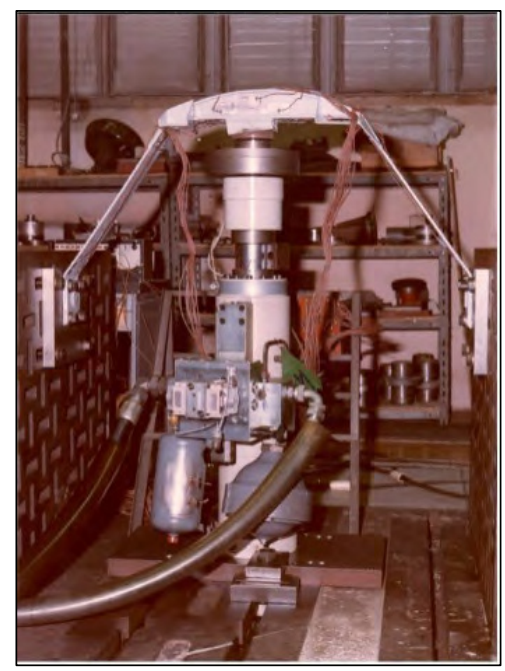

Fig. 14. Fatigue test of a front suspension holder.

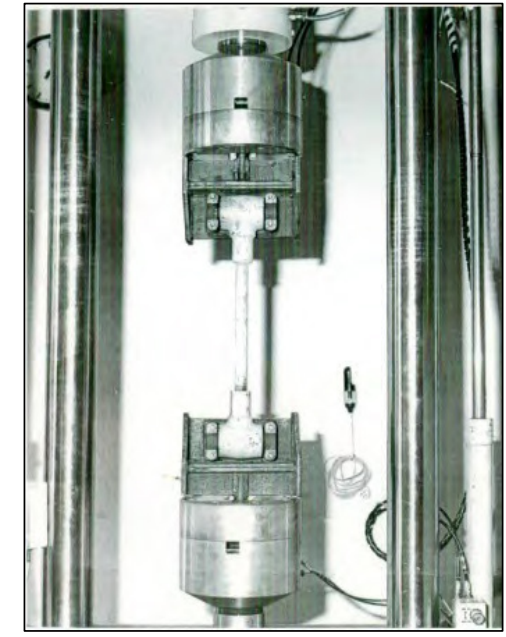

Fig. 15. Fatigue test of a stabilizing guide bar.

\subsection{Activities of Regional Technological Institute}

The Regional Technological Institute is more involved in fatigue life calculations than in the fatigue testing of road vehicles and its parts and components. For these calculations, nCode software is used. The classic calculations of fatigue damage, as well as vibration fatigue, are solved.

The effort is focused on probability dimensioning, taking into account an existing scatter of fatigue properties of materials and random loads of assessed components. In Fig.16, predicted fatigue life distribution functions of a vehicle component are compared to failures that occurred during the vehicle operation.

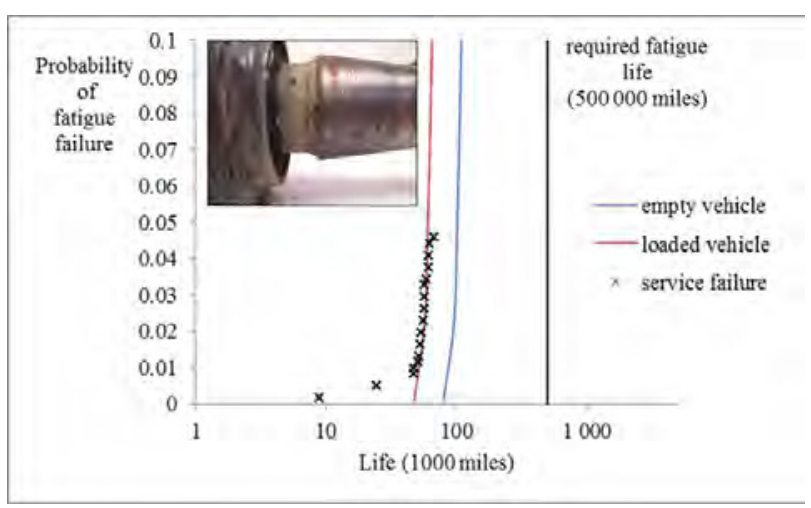

Fig. 16. Predicted fatigue life distribution functions and service failures (radius rod pin).

Figure 17 shows the design of a test rig to be used for multiple testing of welded body profile joints. 


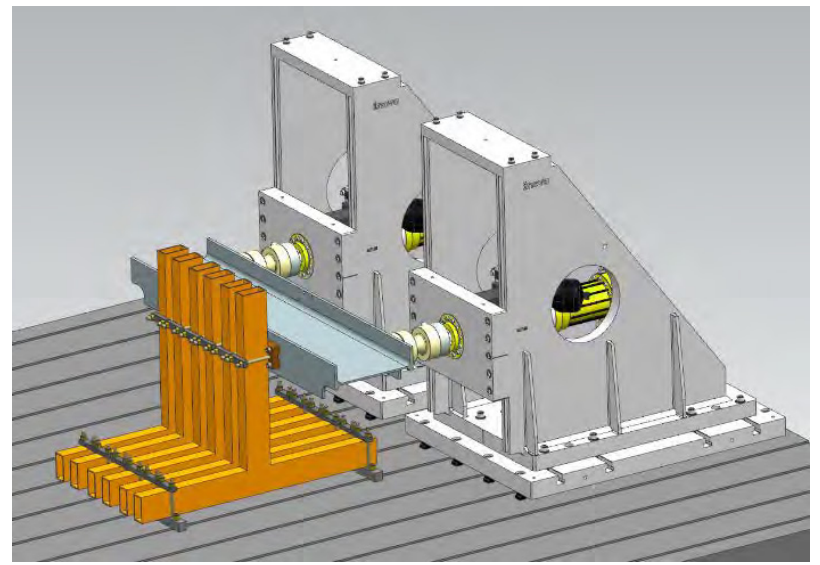

Fig. 17. Predicted fatigue life distribution functions and service failures (radius rod pin).

\section{CONCLUSIONS}

The fatigue tests play a major role in the development of mechanical parts of road and rail vehicles. The contribution demonstrates their variants. The presented examples were prepared and realised in collaboration of producer Skoda Transportation with dynamic testing laboratories at the Research and Testing Institute and the Regional Technological Institute in Pilsen, Czech Republic. The results of these fatigue tests and fatigue life investigations are described in nonpublic testing reports.

The contribution has been prepared under project LO1502 'Development of the Regional Technological Institute' under the auspices of the National Sustainability Programme I of the Ministry of Education of the Czech Republic aimed to support research, experimental development and innovation. 